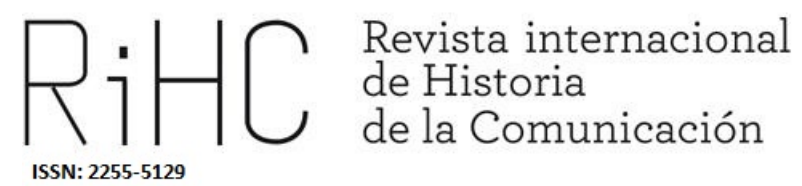

\title{
TINTA FRANQUISTA AL SERVICIO DE HITLER: LA EDITORIAL BLASS Y LA PROPAGANDA ALEMANA (1939-1945)
}

Francoist Ink at Hitler's Service: Blass Publishing House and German Propaganda (1939-1945)

DOI: http://dx.doi.org/10.12795/RiHC.2019.i12.17

Recibido: 08/01/2019

Aceptado: 16/01/2019

Publicado: $15 / 06 / 2019$

Antonio César Moreno Cantano

Universidad Complutense de Madrid, España

antmor03@ucm.es

ORCID (iD) 0000-0003-1008-2831

Mercedes Peñalba-Sotorrío

Manchester Metropolitan University, Reino Unido

penalba-sotorrio@mmu.ac.uk

ORCID (iD) 0000-0002-2774-7556

Como citar este artículo: MORENO CANTANO, Antonio César y PEÑALBA-SATORRío, Mercedes (2019): "Tinta franquista al servicio de Hitler: la editorial Blass y la propaganda alemana (1939-1945)", en Revista Internacional de Historia de la Comunicación (12), pp. 344-369. 
Resumen: La estratégica posición de la Península Ibérica la convirtió en uno de los principales focos propagandísticos del Tercer Reich en el exterior. En este contexto y en el marco de las grandes campañas de propaganda nazi desarrolladas en España, fue clave la política cultural desarrollada por la embajada, que hizo uso de diversas editoriales españolas. Blass, editorial hispana de origen germano, fue una de las más prolíficas en la defensa del nacionalsocialismo. Una labor que no hubiera desempeñado con el mismo éxito sin la ayuda de la política editorial española. De hecho, las actividades de esta editorial confirman no solo la extensión de la red propagandística nazi en España, sino cómo las campañas de propaganda se desarrollaron gracias a la conjunción de la iniciativa propagandística de la embajada, la cooperación de la colonia alemana y la colaboración de Falange. Así mismo, ponen en relieve el carácter profundamente anglófobo de la propaganda alemana en España durante la Segunda Guerra Mundial y arrojan luz sobre el público al que iba dirigida.

Palabras clave: Propaganda de guerra, España, Alemania Nazi, Franquismo, Segunda Guerra Mundial, Falange.

\begin{abstract}
The Strategic position of the Iberian Peninsula made it into one of the main targets for Nazi propaganda abroad. In this regard, the cultural diplomacy of the German embassy in Madrid, which made use of several Spanish publishers, was key to the development of the large Nazi propaganda campaign in the country. Blass, a spanish publisher of german origin, was in this context one of the most prolific in its defence of National Socialism. Its success, however, owed much to the Francoist government's publishing policies. In fact, this publisher's activities show how the development of the Nazi propaganda network in Spain and its subsequent propaganda campaigns developed within a framework marked by the embassy's initiative in propaganda matters, the German colony's cooperation, and Falange's collaboration. At the same time, these activities show the strong Anglophobe character of German propaganda in Spain during the Second World War and shed new light over the audience to which it was aimed.
\end{abstract}

Keywords: War Propaganda, Spain, Nazi Germany, Francoism, Second World War, Falange.

\title{
A modo de presentación: contexto historiográfico y metodológico
}

La estratégica posición de la Península Ibérica en el Mediterráneo y su proximidad a Francia hicieron que el Tercer Reich la convirtiese en uno de sus principales focos propagandísticos en el exterior. Además, sus vinculaciones con Hispanoamérica la convertían en un puente de penetración cultural idóneo para cruzar el Atlántico. Por esa razón, ya en el periodo de entreguerras diferentes organismos alemanes orquestaron complejos planes para la introducción de su propaganda ideológica en territorio español (De la Hera, 2002). El apoyo de Hitler al bando franquista intensificó, como era lógico, este tipo de iniciativas (Whealey, 1989; Leitz, 1996; Collado-Seidel, 2006). El punto 
álgido de estas operaciones se vivió tras el estallido de la contienda mundial, época en la que existió una auténtica "guerra de propagandas" en España, entre las potencias del Eje y las naciones Aliadas (Longerich, 1987; Schneider, 1994; Fernández-Longoria, 2007; Pizarroso Quintero, 2009; Peñalba-Sotorrío, 2012; Moreno Cantano, 2016). La participación activa y decidida del régimen franquista, tanto a nivel político, económico y propagandístico, a favor de su socio germano durante todo este periodo esta fuera de toda duda (García Pérez, 1994; Ros Agudo, 2002 y 2008; Viñas, 2016). Nuestra investigación quiere aportar nuevas pistas sobre este último aspecto, es decir, sobre la política editorial española hacia las producciones culturales nazis que circularon dentro de sus fronteras hasta 1945 (Delgado Gómez-Escalonilla, 2001; Ruiz Bautista, 2005; Bernal Martínez, 2007; Martínez Rus, 2014). No se trataba exclusivamente de libros, sino de meros folletos, opúsculos, hojas sueltas... impulsados por dos relevantes personajes de la Embajada alemana en Madrid, el agregado de Prensa, Hans Lazar, y el agregado cultural, Joseph W. Petersen (Peñalba-Sotorrío, 2016; Janué i Miret, 2001). Personificaremos todas estas iniciativas bajo el paraguas que propició Blass, editorial hispana de origen germano y uno de los principales artífices de toda producción propagandística y cultural en defensa de los valores nazis en lengua castellana. Para ello recurriremos a documentación inédita de gran interés procedente del Politisches Archiv Auswärtiges Amt. La parte referente a los expedientes de censura de la referida editorial, sus contenidos, circulación... la cubriremos a partir del Archivo General de la Administración, en especial los fondos de Cultura y Exteriores. Todo este material se combinará con las obras originales editadas durante los años de guerra mundial, así como con actualizada bibliografía, nacional y extranjera, sobre esta temática. El objetivo último del presente texto es aportar nueva luz sobre la cooperación entre la España franquista y la Alemania hitleriana y, en la medida de nuestras posibilidades, el grado de recepción e influencia de esta índole de escritos entre un tipo específico de lector español (Sevillano Calero, 2000).

\section{La propaganda de la Embajada alemana en Madrid}

La dirección de las campañas de propaganda alemana en España durante la Segunda Guerra Mundial estuvo principalmente en manos de Hans Lazar, agregado de prensa desde $1938^{1}$, quien durante los primeros años del conflicto trabajó en estrecha colaboración con el embajador Eberhard von Stohrer. El diplomático alemán, que ya se

\footnotetext{
${ }^{1}$ Auswärtiges Amt (en adelante, AA) / Politisches Archiv (en adelante, PA)/ Hans Lazar Akt 8.624 y Botschaft Madrid Personalakten / Hans Josef Lazar.
} 
había encargado de organizar campañas de propaganda en España durante la Primera Guerra Mundial (Waddington, 2007: 573-594) ${ }^{2}$, resultaría instrumental en el diseño de nuevas iniciativas de esta índole, una actividad que no solo mostraba claras continuidades con políticas previas al establecimiento del régimen nazi, sino que reafirmaba la importancia de la Península Ibérica no solo por su posición estratégica, sino en virtud de sus relaciones e influencia en otros países, particularmente en América Latina y el Norte de África. Gracias a la habilidad y don de gentes de Lazar y a la diplomática habilidad de Stohrer, el aparato propagandístico nazi creció exponencialmente en España durante los primeros años de la contienda. De hecho, la embajada alemana en Madrid era, en 1941, la más grande del Reich con 42 consulados en total (Ruhl, 1986: 279), Ilegando a ser definida, a su llegada a finales de 1942, por el embajador Hans-Adolf von Moltke como una «hydra de múltiples cabezas» ${ }^{3}$.

En materia de propaganda, Lazar y Stohrer pusieron en marcha una serie de campañas que quedarían complementadas con la política cultural, desarrollada más directamente por Wilhelm Petersen. Esta propaganda fue cambiando en tácticas y objetivos en virtud de la evolución del conflicto, el desarrollo de la política española y la evaluación de las circunstancias y oportunidades que hacían más factible un tipo de propaganda u otra. Así, en los comienzos del conflicto, la propaganda nazi hubo de alejarse del profundo carácter anti-bolchevique que la había caracterizado durante la guerra civil española (Waddington, 2007 y 2012) y pasó a centrarse en controlar, en la medida de lo posible, la narrativa en torno a los orígenes de la contienda. En manos de los propagandistas alemanes, el Tercer Reich se convirtió en defensor y garante del derecho a la neutralidad y Gran Bretaña en potencia imperialista que utilizaba a las naciones europeas como peones en una guerra que había que librar lejos de sus fronteras (Peñalba-Sotorrío, 2012). En este contexto, la propaganda nazi de los primeros años encontró en España, y particularmente en el falangista diario Arriba, un sustrato fértil en el que arraigar. A través de los Boletines de Información de la Embajada Alemana, y de las agencias de noticias, la perspectiva alemana se filtró con facilidad en la prensa española, especialmente en aquellas publicaciones controladas por Falange. De hecho, la estrecha relación de Lazar con el partido único y con otros miembros de importancia del gabinete franquista fue clave en el desarrollo y diseminación de todas estas campañas.

Ya desde su llegada, Lazar había comenzado a estrechar sus vínculos con la prensa española. Así, mientras el Deutsches Nachrichten Büro (DNB) difundía sus noticias a través de la española Fabra desde 1934 (Schulze Schneider, 2004: 59), Transocean (TO), que desde la I Guerra Mundial operaba, de facto, como una rama más del Ministerio de Asuntos Exteriores (Evans, 2010: 216), enviaba las suyas directamente a través de la

\footnotetext{
${ }^{2}$ AA / PA / Botschaft Madrid 716

3 Diario Secreto de Hans Adolf von Moltke, Enero-Marzo 1943, p. 1-2, Archivo General de la Administración (en adelante AGA), Asuntos Exteriores, 82/11809.
} 
recién creada $E F E$, que además recibía su información principalmente de las agencias del Eje: DNB, TO y Stefani (Moreno Cantano, 2008: 120). Por otra parte, utilizaban a las agencias Arco y Faro para difundir breves, comentarios y artículos entre la prensa provincial $^{4}$. También contaban con el servicio de noticias Berliner Briefe que enviaba información a más de 50 cabeceras $^{5}$. Así mismo, Lazar estableció un acuerdo con $A B C$, Informaciones, Madrid y La Vanguardia por el que sus corresponsales en Berlín enviaban sus reportajes directamente a través de la alemana TO, con lo que esperaban poder asegurar un mayor influjo sobre sus noticias y artículos ${ }^{6}$. Además, habría que tener en cuenta que los dos únicos corresponsales con los que contaba EFE en el extranjero trabajaban desde Berlín y Roma y compartían la característica de ser ardientes falangistas (Moreno Cantano, 2008: 120). De este modo, la prensa española se nutría de material pro-alemán por varias vías, especialmente, la embajada, las agencias DNB y TO, y la recepción de artículos a través de los corresponsales asentados en Berlín, de procedencia falangista y claramente influenciados por el ambiente nacionalsocialista.

Lazar mantuvo además estrechas relaciones con los directores de los principales periódicos de Madrid, quienes recibían instrucciones diarias del agregado (Longerich, 1987: 305), así como con los dirigentes de la Delegación de Prensa y Propaganda falangista, Enrique Giménez Arnau, Dionisio Ridruejo, y José María Alfaro (Moreno Cantano, 2008: 127), cuyo nombramiento como subsecretario de prensa y propaganda fue celebrado en la casa que Lazar tenía en Salamanca ${ }^{7}$. Gracias a estos contactos, Lazar logró que se censuraran una serie de obras escritas por autores judíos o dañinas para el Eje (Moreno Cantano, 2008: 259), e incluso que algunas cabeceras anteriormente críticas con Alemania acabaran atenuando su discurso ${ }^{8}$. No es de extrañar, por tanto, que algunos de los argumentos utilizados por la propaganda alemana acabaran haciéndose un hueco en la prensa española.

El agregado de prensa contaba con un presupuesto mensual de 200.000 pesetas, oficialmente orientado a comprar espacio publicitario para empresas alemanas en la prensa española. Sin embargo, la realidad era muy distinta. Tan solo 25.000 pesetas de dicho presupuesto servían a tal objetivo, mientras que el resto del dinero se utilizaba para sobornar a periodistas y escritores españoles (Schulze Schneider, 1994: 374). Un buen ejemplo de esta política fue la publicación del folleto ¿Por qué lucha Alemania? Cómo se ha empujado a Hitler a la Guerra escrito por Federico de Urrutia (bajo el pseudónimo José Joaquín Estrada) (Ruiz Bautista y Barruso Bares, 2011: 194). No en

\footnotetext{
${ }^{4}$ Eberhard Von Stohrer al Auswärtiges Amt, Nr. 6510/39, Madrid, 16/9/39, AA/PA Botschaft Madrid 716.

${ }^{5}$ Eberhard Von Stohrer al Auswärtiges Amt, Nr. 6685/39, Madrid, 10/10/39, AA/PA Botschaft Madrid 788.

${ }^{6}$ Eberhard Von Stohrer al Auswärtiges Amt, Nr. 6510/39, Madrid, 16/9/39, AA/PA Botschaft Madrid 716.

${ }^{7}$ AA / PA / Botschaft Madrid 788.

${ }^{8}$ AA / PA / Botschaft Madrid 788.
} 
vano, Urrutia se convertiría más tarde en uno de los principales colaboradores del Gran Plan, la mayor empresa de propaganda diseñada por la embajada durante este periodo.

El aumento de la propaganda aliada en la Península Ibérica registrado en 1941, junto con la entrada de Estados Unidos en la guerra y el estancamiento en el frente del Este, llevó a Lazar y Stohrer a diseñar y lanzar su campaña de propaganda más ambiciosa, el conocido como Gran Plan, que se pondría en marcha en enero de 1942. Todas las publicaciones, carteles, folletos y bandos diseminados en el marco de la misma aparecían como publicaciones anónimas o independientes, en ningún caso conectadas con la embajada alemana, aunque de vez en cuando reconociendo un origen falangista. De hecho, el Gran Plan se apoyaba, más que ninguna otra misión de esta naturaleza, en la estrecha colaboración con Falange, dado que tanto la interceptación de propaganda enemiga, como la diseminación de nuevos materiales estaba en manos del partido único (Dankelmann, 1969; RUHL, 1986: 41-42; Schulze Schneider, 1994: 371-386). Por supuesto, dado el carácter contra-propagandístico del proyecto, la mayor parte de los temas tratados venían determinados por la propaganda aliada. No es extraño, por tanto, que estas publicaciones se centraran especialmente en destacar las debilidades de los aliados, en aumentar la propaganda religiosa que buscaba convertir al Tercer Reich en adalid del cristianismo, o en cantar las bondades del Nuevo Orden. Mensajes más fácilmente diseminables en una nueva etapa del conflicto marcada por el renovado enfrentamiento entre el Tercer Reich y la Unión Soviética. El nuevo estado de cosas permitía a los propagandistas recuperar y revitalizar todos aquellos argumentos antibolcheviques que tan efectivos habían sido durante la guerra civil y utilizar la alianza entre los aliados y la Unión Soviética para denunciar a las potencias occidentales como anticristianas y antieuropeas. 


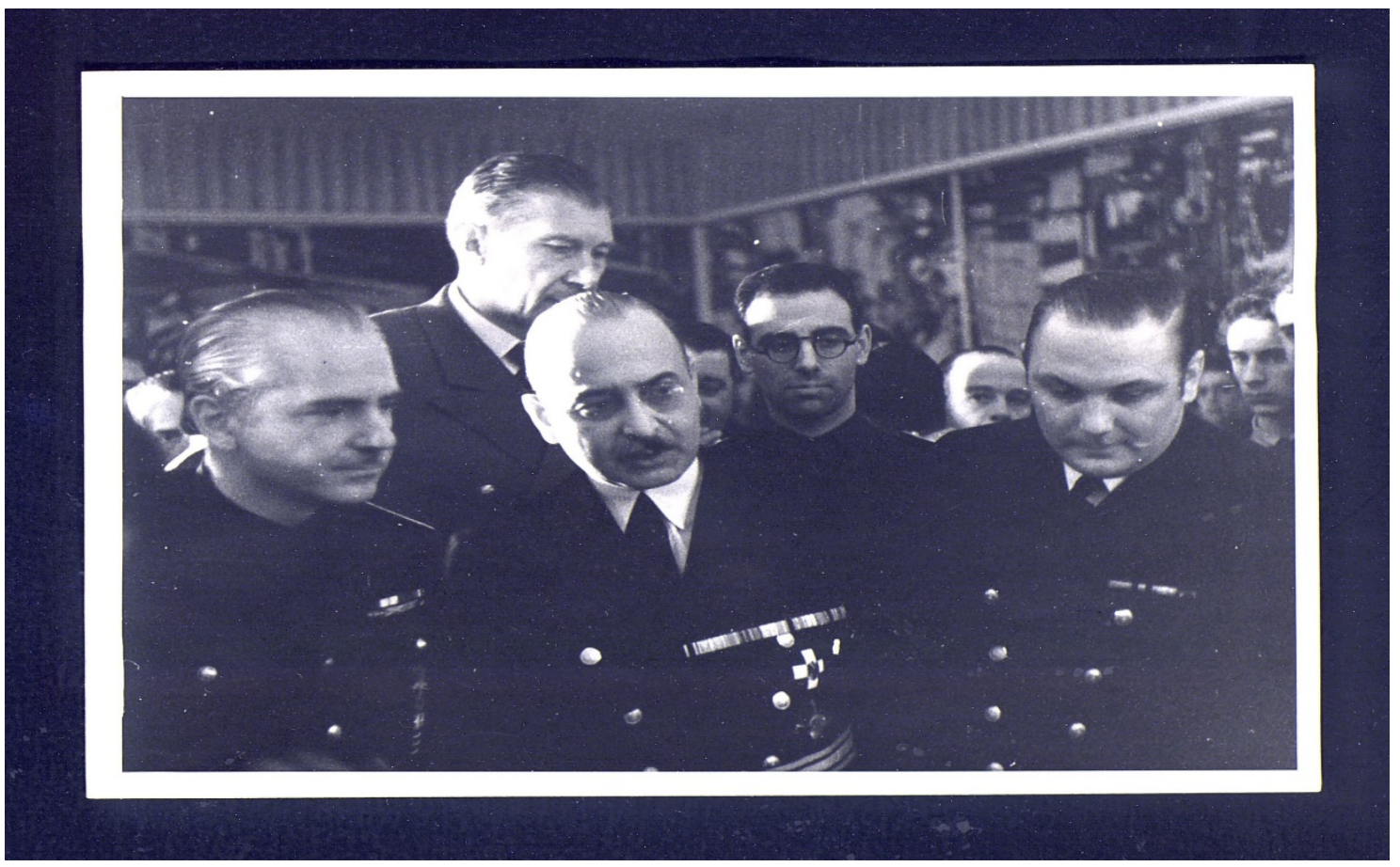

Figura 1. Los máximos jerarcas de la propaganda española y germana en marzo de 1941, durante la Exposición de la Prensa Alemana en Madrid. De izquierda a derecha, el ministro de Exteriores, Serrano Suñer; el agregado de Prensa, Hans Lazar; sobre él, embajador Von Stohrer; el subsecretario de Prensa y Propaganda, Antonio Tovar; y el jefe de Prensa Extranjera del Ministerio de Exteriores alemán, Paul Schmidt. Fuente: MECD, AGA, Cultura, caja 3799.

Todas estas campañas coexistieron, por supuesto, con una práctica constante por parte de la embajada alemana en Madrid, la de monitorizar la prensa española y velar por la inclusión de noticias favorables al Eje. Una actividad tanto más compleja cuando se incluía a los países latinoamericanos en el marco de actuación, y se tenía en cuenta que aquello que se publicaba en España tenía eco en América Latina y viceversa. Esta problemática se insertaba en un marco de continuidad con prácticas ya establecidas durante la Primera Guerra Mundial, cuando España se convirtió en un instrumento para mantener la neutralidad latinoamericana y aumentar la influencia alemana en la zona, unas actividades que cristalizaron en la fundación del Instituto Iberoamericano de Berlín en 1929 (Albes, 1996: 244-248; Delgado Gómez-Escalonilla, 1992: 148; De la Hera Martínez, 2002: 106). En el contexto de la Segunda Guerra Mundial, estos objetivos no cambiaron, pero su consecución se hizo más compleja, ya que era determinante mantener también la actitud benevolente de España hacia el Eje en Europa, que se podía ver influida por la evolución de la política alemana en América Latina.

En este contexto, la figura de Blass Mayer se sitúa en la intersección entre las directrices diseñadas por Stohrer y Lazar y el desarrollo de la política cultural de Petersen. Es más, Blass Mayer se yergue también como exponente de la instrumentalización de la colonia alemana para la diseminación de la propaganda nazi y la cultura nacionalsocialista. La editorial Blass no solo estaba encargada de publicar algunos de los boletines de 
información de la embajada, que aparecían tres veces por semana, con una tirada de unos 45.000-60.000 ejemplares (Schulze Schneider, 1995: 202), sino que fue instrumental en la traducción y diseminación de una serie de obras nacionalsocialistas, directamente importadas desde Alemania, que buscaban colaborar a la integración de España en el Nuevo Orden europeo. Objetivo que también perseguían otras editoriales de origen alemán amparadas por la embajada alemana y controladas por el Ministerio de Asuntos de Exteriores de Berlín, como la editorial Persiles o Nueva Época, ambas establecidas en 1942 (Longerich, 1987: 271), en el marco de desarrollo del Gran Plan.

OSLO. - La noticia de la firma del Tratado de paz entre Finlandia y la U. R. S. S., ha causado una impresión muy favorable. Se espera que después de la terminación del conflicto, la Unión Soviética intensificará sus relaciones comerciales con Alemania. El "Morgenposten" se felicita de que Finlandia haya firmado el Tratado de paz, que hace que este pais conserve su independencia y asegure la paz en todo el Norte de Europa. - D. N. B.

Blass, S. A. - Madrid:

\section{La editoria Blass y la propaganda germanófila}

El nombre de Joseph Blass Mayer ha permanecido en el anonimato en los principales estudios que han analizado la política editorial y propagandística española entre 1939 y 1945, siendo mencionado únicamente en su faceta de tipógrafo en décadas previas (Martínez Martín, 2001: 381; Fontes y Menéndez, 2004: 402). Nunca se ha indagado sobre la figura de este ciudadano alemán, responsable de la impresión y edición de los principales folletos y opúsculos de tono anglófobo que circularon por territorio español, siendo parte activa de la "guerra de propagandas" que se dilucidó en la Península Ibérica. En el presente apartado aportaremos luz sobre su biografía hasta el inicio de la contienda mundial. Seguidamente, analizaremos sus conexiones con la embajada germana en Madrid, así como los principales títulos que editó, poniendo el interés en el contexto en que se realizaron, el contenido de los mismos, así como los destinatarios entre la ciudadanía española. 


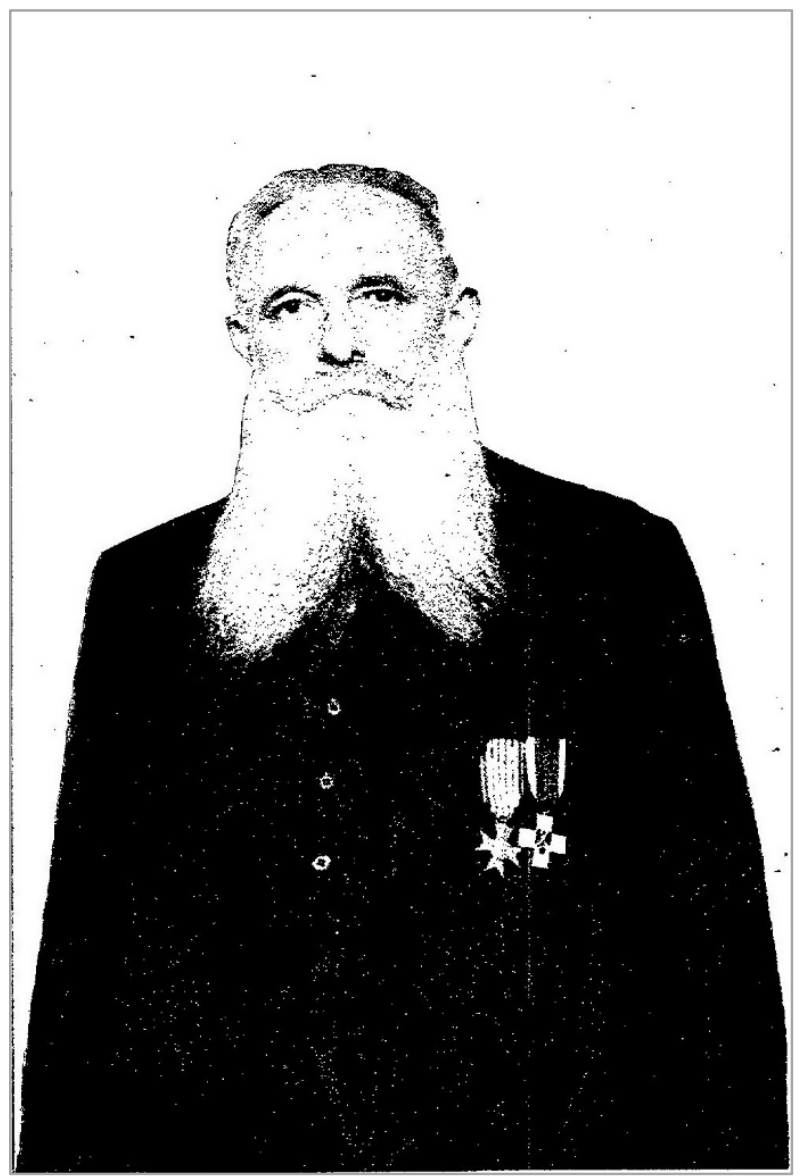

Figura 3. Joseph Blass Mayer en 1936. Fuente: $A B C$

Considerado como el "primer hispanófilo en nuestra Patria" ${ }^{9}$, nació en Munich el 9 de mayo de 1873. Vino a España el 6 de marzo de 1899, reclutado en persona por el propio director de $A B C$, Torcuato Luca de Tena, para desempeñar el cargo de Jefe de máquinas e instructor para la revista Blanco y Negro (Olmos, 2002: 45), a la que estuvo adscrito hasta $1903^{10}$. A partir de esa fecha constituyó su propia imprenta, adquiriendo un gran éxito gracias a sus producciones de gran calidad y renombre como fueron la revista $E I$ Cuento Semanal. Fue el encargado de cerrar el Primer Congreso Nacional de las Artes del Libro de Barcelona (octubre de 1912), en el que dio muestras de gran patriotismo con gritos de "viva España", que fueron contestados con otros a Alemania ${ }^{11}$. Representó al gremio de tipógrafos peninsular en la Exposición Internacional de las Artes Gráficas de Leipzig de $1914^{12}$. Su relación con la colonia germana, además, era muy intensa, como prueba su participación en la inauguración de la Sociedad Gimnástica Alemana de

\footnotetext{
9 “Reproducción de las mejores pinturas religiosas de España”, Tierra Charra, 24 de noviembre de 1929.

${ }^{10}$ AGA, Exteriores, caja 10150. Escrito al Ministerio de Asuntos Exteriores, Dirección General de Política Económica, Servicio de Bloqueo de Bienes Extranjeros, expediente 62, 19 de agosto de 1948.

11 “Las Artes del Libro” La Correspondencia de España: diario universal de noticias, 19 de octubre de 1912.

${ }^{12}$ Federación Nacional de las Artes del Libro, n. 4, mayo, 1914, p. 13.
} 
Madrid en 1915 , en la que entonó varias canciones populares ${ }^{13}$. Por su actuación durante la Primera Guerra Mundial, Alemania le concedió la Cruz de Hierro de tercera clase $^{14}$.

En los años treinta alcanzó una gran fama mediante la creación de la técnica del "Artóleo", que permitía realizar reproducciones de enorme calidad de pinturas al óleo, tales como La maja desnuda, Las hilanderas o Las meninas, expuestas con gran afluencia de visitantes en diferentes salas de Madrid ${ }^{15}$. En 1933, uno de sus hijos, José María Blass König, aparecía como profesor de tipografía -siguiendo sus pasos- en la Escuela de Periodismo de $E$ Debate $^{16}$, y ejerciendo como propagandista de la Asociación Católica Nacional de Propagandistas en los años cincuenta ${ }^{17}$. Y en 1936, pocos meses antes de la Guerra Civil, como recompensa a su larga trayectoria como impresor y editor, así como por sus labores sociales, recibía la Gran Cruz de Beneficiencia por parte de la Embajada alemana $^{18}$. Sus éxitos le granjearon el apoyo y reconocimiento de las autoridades españolas como del cuerpo diplomático alemán, los cuales serían claves para entender su actividad propagandística tras el inicio de la conflagración mundial. Sin embargo, tampoco le faltaron "enemigos", que se creó a lo largo de toda esta trayectoria y que, sin lugar a dudas, también determinaron su posicionamiento futuro. Entre 1909 y 1931 sufrió en su empresa tres huelgas y, lo más grave, un intento de asesinato por parte de varios trabajadores (entre los que se incluía un secretario del Partido Comunista) en 1921. Se le reprochaban sus bajos salarios y sus despidos masivos ${ }^{19}$. Más tarde, durante el conflicto español, dos de sus hijos, el referido José María y su hermano Enrique, fueron incomunicados -posiblemente por desarrollar labores de espionaje $y$ quintacolumnismo en la capital- en la Prisión Provisional de Madrid, número 3, por orden del Departamento Especial de Información del Estado (DEDIDE), germen del futuro Servicio de Información Militar (SIM) ${ }^{20}$. Posteriormente fueron traslados a Valencia, donde acabaron siendo canjeados por mediación de la diplomacia alemana en $1938^{21}$.

\footnotetext{
13 "Nuevo campo de deportes", Gran Vida, 1 de enero de 1916.

14 “Ha muerto don José Blass, maestro de las Artes Gráficas”, $A B C, 16$ de mayo de 1957.

15 "Exposiciones y noticias artísticas", $A B C, 21$ de enero de 1931.

16 "Escuela de Periodismo de El Debate", Contemporánea (Valencia) octubre de 1933, p. 150.

${ }^{17}$ A.C.N. de P., 1 de enero de 1960, p. 7.

18 “Distinción al gran artista D. José Blass”, Blanco y Negro, 9 de febrero de 1936.

19 "La Huelga de la Gaceta...", La Correspondencia de España, 20 de enero de 1909; ABC, "Caso inaudito. Procedimientos socialistas", 5 de abril de 1914; El Debate, "Madrid", El Debate, 8 de julio de 1921; y "La huelga de los obreros de las artes gráficas", La Voz, 19 de enero de 1931.

${ }^{20}$ Archivo del Ministerio del Interior, signatura 44.466. Expediente de José María Blass König, 1937.

${ }^{21}$ AGA, Exteriores, caja 10150. Escrito al Ministerio de Asuntos Exteriores, Dirección General de Política Económica, Servicio de Bloqueo de Bienes Extranjeros, expediente 64, 19 de agosto de 1948.
} 
La familia al completo regresó a Madrid tras la victoria franquista, siendo los responsables de la publicación, en edición facsímil, del Parte oficial de guerra del 1 de abril de $1939^{22}$. Su vinculación con la embajada germana, a partir de la Segunda Guerra Mundial, se intensificó mediante la publicación -como se apuntó en páginas anterioresde numerosos folletos y panfletos abiertamente beligerantes contra Inglaterra, en especial, a partir del verano-otoño de 1940. La fecha no obedecía a la casuística, no en vano tras la caída y rendición de Francia se puso en marcha por parte de la Wehrmacht la Operación León Marino (Directiva n. 16 de Hitler), que pretendía invadir Gran Bretaña (Forczyk, 2016), la cual se acompañó de una intensa campaña propagandística anglófoba dentro y fuera del Tercer Reich (Hayward, 2016). Aunque la anglofobia no era una novedad en los materiales propagandísticos destinados a España, ciertamente la evolución del conflicto intensificó los ataques contra Inglaterra. Una actitud que encontraría su apoyo en la Península, puesto que la campaña coincidía además con la declaración de "no-beligerancia" del régimen franquista, en un claro movimiento geopolítico a favor del Eje, acompañado de duros ataques propagandísticos a Inglaterra. El 31 de mayo de 1940, el periodista Manuel Aznar publicaba en Arriba el artículo "Gibraltar, honor y deber de los españoles", en el que reclamaba de forma encendida la devolución del peñón: "Gibraltar es de España, que nadie puede retenerlo sin incurrir en delito de despojo" (Fernández-Longoria, 2007: 195). Al día siguiente, coincidiendo con la llegada del embajador Samuel Hoare, se produjo una manifestación de estudiantes y falangistas contra la Embajada británica (Moradiellos, 2005: 139).

En este ambiente, los censores de la Delegación Nacional de Propaganda recibieron, en la primera semana de octubre, cuatro ejemplares procedentes de la embajada germana en Madrid focalizados en esa temática: Religión, arma del imperialismo inglés; Caza del zorro o pan; Inglaterra y el continente europeo; y El Calvario de Chipre. Estos títulos eran, en la mayoría de casos, traducciones al castellano impulsadas por la Deustche Informationsstelle (Oficina de Información Alemana) del Ministerio de Exteriores germano, y en concreto del Jefe de Prensa Extranjera, Paul Schmidt. Se adscribían a la serie "England Ohne Maske", a la que pertenecían obras, también de 1940, como England und die Buren, Englands gewaltpolitik am Nil o Englands regiment in Pälestina, sumamente críticas con la política colonial británica: "Chipre es un ejemplo de la falta de interés de los plutocráticos gobernantes ingleses" (Embajada Alemana, 1940: 25). El objetivo del Deutsche Informationsstelle era utilizar editoriales "independientes" (caso de Blass, Rubiños o España ${ }^{23}$ ) de otros países para ocultar sus orígenes y evitar las

\footnotetext{
22 “Parte de la Victoria. Una obra de mérito", Heraldo de Zamora, 18 de marzo de 1940.

${ }^{23}$ Publicó en la década de los 40 nueve títulos de propaganda procedentes de "organismos oficiales alemanes" y cuatro de autoría española, como la referida de Joaquín Estrada, o las de Félix Cuquerella ( $L a$ guerra de hoy. iiEuropa resucita!!), Juan Agero (Así fue posible. Antecedentes de la segunda crisis europea en el siglo XX) y Alfonso Castro. Álvarez Chillida, 2002, p. 383. Encargos a las editoriales Rubiños, España y Blass, AA / PA / Botschaft Madrid, Sammlung der Berichte, 761.
} 
cortapisas del bando Aliado. Incluso, se ocultaba -en determinados casos- la autoría o la significación del título al ser traducido a otras lenguas extranjeras ${ }^{24}$.

Los tres textos nombrados fueron revisados y autorizados en solo una semana, lo que resulta sumamente sorprendente (solo de manera funcional, no ideológica), pues escritos como Alas germanas sobre Europa ${ }^{25}$, de Spectator (pseudónimo del periodista y corresponsal de guerra Alberto Martín Fernández), fue retenido un año entero, de enero de 1940 a enero de 1941 , en las oficinas de la censura española ${ }^{26}$. ¿La razón? No disponía de fondos para su publicación y, quizás, no era el contexto geopolítico adecuado. Los expedientes de censura, en los que nos apoyaremos en este apartado, son una herramienta muy útil para conocer el parecer franquista sobre este tipo de propaganda. De Religión, arma del imperialismo inglés se señalaba que denunciaba la "farisaica conducta del Imperialismo, hipócritas procedimientos de la pérfida Albión" y de Inglaterra y el continente europeo, en el apartado "otras observaciones" se explicaba que "trae datos históricos para concluir afirmando que el cáncer de Europa es el imperialismo británico". Fueron revisados por el censor Enrique Romeu (sustituido a principios de 1941 junto al resto de personal de su sección, en su caso por no pertenecer al Partido, pese a ser considerado "el mejor de todos los censores") (Ruiz Bautista, 2008: 55) y se editaron 15.000 ejemplares de cada uno de ellos, siendo la editorial Blass responsable de su impresión y venta ${ }^{27}$. Desconocemos el pago total que recibió esta empresa por sus servicios a la embajada, pero sí sabemos que, en 1941, por la edición de 3.000 ejemplares de Inglaterra a través de la crítica inglesa recibió 2.834 ptas. y 415 ptas. por la impresión de 25.000 copias de un anexo para el Boletín de Información titulado "La libertad de los mares" ${ }^{28}$. La diferencia de precio estriba en que los anexos solían ser muy breves, entre dos y cuatro páginas. Claramente, la empresa resultaba rentable, como lo prueba la gran cantidad de títulos que editó a posteriori.

\footnotetext{
${ }^{24}$ TAUBE, 1944, pp. 86-90.

${ }^{25}$ Se trataba de una recopilación de fotografías sobre las principales acciones del Ejército Alemán del Aire en los primeros años de la guerra, acompañadas cada una de ellas con una breve descripción. Como destacó la censura franquista, "el libro tiene su más clara intención en llevar al ánimo del lector la conciencia de hallarnos ante el estrepitoso fracaso de un mundo viejo y el amanecer de un nuevo orden". Se insistía, como en tantas otras ocasiones, que la contienda en España había constituido el primer paso en este camino. En su interior, Spectator elogiaba a Hitler ("Caudillo altivo y patriota") y el resurgir alemán tras el Tratado de Versalles ("una gran nación continental que en 1918 fue brutalmente aniquilada aunque no vencida- en nombre de la faramalla democrática y justiciera"). El autor no tenía ningún reparo en mostrar y vanagloriarse de "la exactitud y precisión de los bombardeos alemanes" o de los "efectos de los ataques sobre las grandes ciudades alemanas". AGA, Cultura, caja 6602, expediente 268, enero de 1940; y SPECTATOR, 1941, p. 109.

${ }^{26}$ Véase, AGA, Cultura, IDD. 050.000, expedientes de censura. Publicaciones de la editorial Blass.

${ }^{27}$ AGA, Cultura, caja 6575, expedientes 965 y 960.

${ }^{28}$ Heberlein al Ministerio de Asuntos Exteriores en Berlín, 20 de octubre de 1941. AA / PA / Botschaft Madrid, Sammlung der Berichte, 761. Petersen al Ministerio de Asuntos Exteriores en Berlín, 3 de noviembre de 1941. AA / PA / Botschaft Madrid, Presselenkung 757.
} 


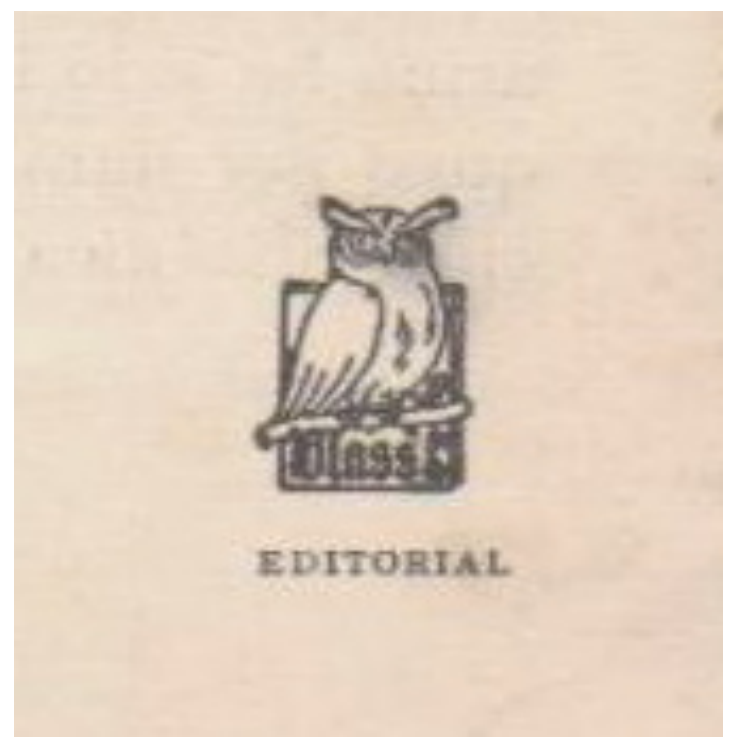

Figura 4. Logotipo de la Editorial Blass

Blass también se encargó de imprimir otras obras, de contenidos muy similares, en consonancia con otras editoriales alemanas asentadas en Madrid, como el caso de Rudolf Kadner Librero ${ }^{29}$, sita en la calle Serrano, número 17. Nos referimos a la obra El espacio vital, de Gerhart Jentsch, traducción directa del alemán de Lebensraum, calificada de valor literario "excelente" y cuya "publicación juzgamos conveniente" ${ }^{30}$.

A partir de la aparición de estos volúmenes aparecieron, de forma continua e ininterrumpida hasta el verano de 1942, fecha en la que Serrano Suñer sale del Ministerio de Exteriores, nuevos títulos que atacaban desde todas las facetas imaginables (política, económica, religiosa...) a Gran Bretaña, en línea con las directrices establecidas para la propaganda en España desde el Referat Inland del Ministerio de Asuntos Exteriores en Berlín. El anticomunismo aparecía aquí como un tema secundario, siempre ligado a la crítica a Estados Unidos y sobre todo a Inglaterra, a quien se acusaba de querer debilitar a España apoyando a los exiliados, tanto de izquierda como monárquicos. El mensaje marcado por los propagandistas alemanes era claro, la anticatólica y pro-bolchevique Inglaterra quería convertir la Península Ibérica en punta de lanza en su lucha contra Alemania ${ }^{31}$. Actividades y directrices que ponen en evidencia que la propaganda nazi en España fue muy activa previamente a la puesta en marcha

\footnotetext{
${ }^{29}$ Como dato de esta beneficiosa relación económica con la embajada alemana, las autoridades norteamericanas señalaron en 1945 que el referido empresario recibió pagos por sus trabajos de propaganda que sobrepasaron las 100.000 pesetas. National Archives and Records Administration (en adelante, NARA), M1922. Records of the External Assets Investigation Section of the Property Division, OMGUS, 1945-1949. "Expediente de Rudolf Kadner".

${ }^{30}$ AGA, Cultura, caja 6528, expediente 984, 6 de mayo de 1940.

${ }^{31}$ Standardthesen und Richtlinien für die Deutschen Auslandspropaganda, Standardthesen für Spanien, 14 Februar 1942, AA / PA / R99474.
} 
del Gran Plan, coincidiendo -en lectura interna- con la época de máximo poder de Falange en las estructuras culturales e informativas.

Durante el mes de octubre de 1940, dos de los folletos mencionados de la serie "England Ohne Maske", fueron traducidos y editados por Blass con la denominación de La política inglesa de violencia en el Nilo y El dominio inglés en la India. La primera de ellas, de la que se editaron dos mil ejemplares, fue obra de Paul Schmits Kairo, periodista alemán especialista en temas africanos que había trabajado para importantes diarios nazis como el Völkischer Beobachter (Rubin y Schwanitz, 2014: 85). La segunda, responsabilidad de Reinhard Frank, jurista de prestigio internacional, utilizaba un lenguaje cargado de los más oscuros apelativos hacia los métodos de ocupación empleados por los británicos en Asia: "la India era un paraíso... hasta que llegaron los ingleses. Porque lo que hoy encontramos en la India es un país donde no hay más que sufrimiento inútil, hambre..." (Frank, 1940: 11). No se trata de desgranar estas obras una por una, sino que pondremos la atención en apuntar aquellos conceptos o argumentos que se repetían de manera mecánica en el conjunto de ellas. Y, sobre todo, conocer el sentir de los censores franquistas en las más representativas.

Antes de finalizar ese año, en el mes de noviembre, y en una línea ideológica casi idéntica a los folletos recién nombrados, se editaron Inglaterra, traficante de esclavos, de Ernest A. Olbert (pseudónimo del profesor Konrad Oehlrich) ; y Humanidad británica frente a indefensos, de Arthur Finck, "un folleto revelador de los malos tratos dados a los prisioneros alemanes en Inglaterra durante la guerra mundial" ${ }^{32}$. En todos estos escritos, y como ha puesto en evidencia Gerwin Strobl, salían a relucir muchos de los prejuicios y percepciones críticas al modelo colonial británico, el mismo que en los años 30 había sido admirado como modelo para conseguir el Lebensraum y que ahora se empleaba como tema propagandístico negativo (Strobl, 2000). La postura española comulgaba punto por punto con la de sus homólogos nazis, pues se consideraba que este género de obras "demuestra con profusión de documentos los aspectos repugnantes de ese bajo oficio de traficar con hombres que los ingleses hacían a gran escala" ${ }^{13}$.

A lo largo de los dos años siguientes, se publicaron casi una decena de folletos centrados en los aspectos económicos de la guerra y la posición de Inglaterra en la misma, incidiendo en su política de bloqueo y aislamiento de determinados países. El tema era de especial interés para los jerarcas falangistas, no en vano el Reino Unido desde el inicio de la guerra había creado un sistema de bloqueo comercial que limitaba las exportaciones españolas al Eje ("The Proclaimed and Statutory Lists") (Marquina, 2014: 100-101), y que había sido duramente criticado en los boletines de información de la

\footnotetext{
${ }^{32}$ AGA, Cultura, caja 6594, expediente 778, 26 de noviembre de 1940.

${ }^{33}$ AGA, Cultura, caja 6592, expediente 573, 16 de noviembre de 1940.
} 
embajada desde el comienzo del conflicto (Peñalba-Sotorrío, 2013). En julio de 1941, el legado de embajada Heyden-Rynsch informaba a Berlín de la publicación y distribución en el área de España, Marruecos español y Fernando Poo, de 186.000 folletos y hojas informativas en los dos meses previos, entre ellos algunos de los publicados por la editorial Blass. Así, se distribuyeron 2.000 ejemplares de La Irlanda del Norte de Ernest Falk, 5.000 de Los judíos en Inglaterra de Erich Zander, 3.000 de Inglaterra a través de la crítica inglesa y otros folletos como Los fines de la guerra en Inglaterra o Potencias occidentales destructoras de la neutralidad ${ }^{34}$. Sin embargo, llama la atención que en la editorial Blass no apareciese ningún escrito contra el comunismo internacional, máxime cuando el 22 de junio de 1941 se iniciaba la Operación "Barbarroja", de invasión de la Unión Soviética por parte de la Wehrmacht y sus aliados. Este hecho nos indica que esa tarea propagandística recaería en otras entidades o medios ${ }^{35}$, como bien pudiera ser la propia prensa española a través de sus numerosas consignas anticomunistas.

A finales de 1942 se animaba a los directores de los principales diarios del país a escribir editoriales que reflejasen la siguiente idea: "Para los españoles no puede haber duda; el dilema Alemania o la URSS es exactamente igual que el dilema de 1936, o la victoria de Franco o la victoria del Frente Popular. El triunfo de Alemania nos pone a salvo..." ${ }^{13}$ Paralelamente, algunos opúsculos de Blass que descalificaban al presidente norteamericano, F.D. Roosevelt, como Entre el Támesis y el Potomac, autorizados en un principio por los organismos propagandísticos falangistas (como era la Vicesecretaría de Educación Popular), fueron retirados de manera fulminante por órdenes del nuevo ministro de Exteriores, el conde de Jordana. En la nueva política de neutralidad que se inauguró tras la salida de Serrano Suñer, juicios tales "¿cómo se atreve el Sr. Roosevelt a decir que él garantiza la prosperidad económica del mundo, él, que está al acecho, como un salteador de caminos...?"137, no eran vistos con buenos ojos por los nuevos rectores de la política internacional franquista.

En este punto, debemos detenernos en las obras antibritánicas que editó en 1941 sobre la temática económica y en las circunstancias en las que se impulsaron. Ese año fue testigo de la intensificación de la colaboración cultural con el Tercer Reich, ya fuese

\footnotetext{
${ }^{34}$ Heyden-Rynsch al Ministerio de Asuntos Exteriores en Berlín, 8 de Julio de 1941; Heberlein al Ministerio de Asuntos Exteriores en Berlín, 20 de octubre de 1941. AA / PA / Botschaft Madrid, Sammlung der Berichte, 761.

${ }^{35}$ La Embajada germana publicó en esas fechas varias hojas de propaganda, con llamativos montajes fotográficos y textuales, como Dios entre los bolcheviques o Los Sin Dios, en los que a la par que se arremetía contra el ateismo soviético se criticaba el beneplácito británico con esta política de persecución religiosa: "Quien hoy grite viva Churchill es que aspira a que parte de Europa corra la misma suerte que corrió Rusia, su Catedral de Smolensko antes de ser nuevamente abierta al culto y las demás iglesias rusas, transformadas en salas de baile. A un lado está Europa, salvaguardia de nuestra civilización milenaria y al otro, los conjurados contra todos nuestros bienes sacrosantos".

${ }^{36}$ AGA, Cultura, caja 77. Consigna de prensa anticomunista, 28 de septiembre de 1942.

${ }^{37}$ AGA, Cultura, caja 6805, expediente 937.
} 
mediante la constitución de la Asociación Hispano-Alemana; del Instituto de Cultura Alemán (Janué i Miret, 2001: 820-821); o la celebración de exposiciones como la de "objetos de culto donados por Alemania"; la de la "Prensa Alemana"; o la de "Dibujos sobre la nueva Europa continental" 38 . Como parte de esta "colaboración" culturalpropagandística, Blass, por mediación de la Embajada germana y el visto bueno de la censura española, se editaron folletos como La liquidación del prestigio económico mundial de Inglaterra en la guerra, en la que la economía nazi era descrita como un sistema "respetuoso con las particularidades étnicas y la autonomía política de los pueblos" (Kiesewetter, 1941: 13) ; o La alimentación en Europa, de Herbert Backe, subsecretario de Alimentación del Reich, que arremetía contra "el intento de vencer a Alemania por hambre"39.

\begin{tabular}{|c|c|c|c|c|}
\hline Titulo & Nombre & Expe_no & F.Entrada & F.Resolución \\
\hline Inglaterra traficante de esclavos & Olbert, Ernest A. & 573 & $12-11-1940$ & $16-11-1940$ \\
\hline Los bloqueadores bloqueados & Anónimo & 462 & 21-01-1941 & 22-01-1941 \\
\hline Las autopistas del reich alemán & Pilug, Hans & 722 & $10-02-1941$ & $11-02-1941$ \\
\hline $\begin{array}{l}\text { La política de la Gran Bretaña en los últimos } \\
\text { años }\end{array}$ & Laurie & 49 & 03-03-1941 & 10-03-1941 \\
\hline El bloqueo inglés contra Europa & $\begin{array}{c}\text { Sección Prensa de la } \\
\text { Embajada de } \\
\text { Alemania }\end{array}$ & 85 & 04-03-1941 & 06-03-1941 \\
\hline Empresas modelos de Alemania & $\begin{array}{l}\text { Sección Prensa } \\
\text { Embajada }\end{array}$ & 143 & 08-03-1941 & $12-03-1941$ \\
\hline Una economía sin crisis & Uterman, Wilhelm & 214 & 13-03-1941 & 17-03-1941 \\
\hline Nuevas materias primas alemanas & Dorn, Doctor Kael & 556 & $12-04-1941$ & $15-04-1941$ \\
\hline $\begin{array}{c}\text { Liquidación del prestigio económico mundial } \\
\text { de Inglaterra en la guerra }\end{array}$ & Kiesewetter, Bruno & 555 & $12-04-1941$ & $15-04-1941$ \\
\hline Los fines de la guerra en la Gran Bretaña & Zander, Enrich & 732 & $24-06-1941$ & $28-06-1941$ \\
\hline Hambre en Inglaterra & Hafner, Otto Philipp & 173 & 21-07-1941 & $26-07-1941$ \\
\hline La alimentación en Europa & Backe & 689 & 20-01-1942 & $22-06-1942$ \\
\hline Ordenación de los mercados & Dittmer, Hans & 24 & $12-06-1942$ & $25-09-1942$ \\
\hline El humanismo de la guerra británica & Hartig, Paul & 718 & $20-07-1942$ & $25-09-1942$ \\
\hline Los trabajadores españoles en Alemania & Embajada Alemana & 706 & $23-12-1942$ & $15-02-1943$ \\
\hline Economía de Europa después de la guerra & Schmidt, $\mathrm{H}$. & 9 & 02-01-1943 & $15-02-1943$ \\
\hline
\end{tabular}

\footnotetext{
38 "La gran exposición de objetos de culto para las Iglesias españolas", ABC, 22 de febrero de 1941; "La Exposición de Prensa Alemana", $A B C, 11$ de marzo de 1941; y "Arte y artistas. Dibujos sobre la nueva Europa continental", $A B C, 11$ de octubre de 1941.

${ }^{39}$ AGA, Cultura, caja 6792, expediente 689, 21 de enero de 1942.
} 
En 1942, el número de publicaciones de este tipo disminuyó significativamente, aunque seguían apareciendo algunas de las obras distribuidas por Blass, como la mencionada La alimentación en Europa o Los fines de Inglaterra en la guerra en los informes de la Embajada alemana en Madrid, que aún publicaba unos 242 folletos y revistas diversos, tanto en alemán como en español, en la primera mitad de $1942^{40}$. El último folleto de propaganda publicado por Blass fue Economía de Europa después de la guerra, en enero de 1943, una época en la que se sucedían las derrotas de las armas germanas (invasión aliada en el Norte de África, triunfo soviético en la batalla de Moscú, avance angloamericano tras la batalla del Alamein, retroceso en Stalingrado). A partir de ese momento, pese a que seguía en plena vigencia el Gran Plan, Blass dirigió su atención a otro tipo de publicaciones, más en su línea tradicional, previa a la contienda mundial, como podían ser los libros de literatura o de tendencia religiosa; reeditando clásicos del Siglo de Oro; incluso abordando manuales de carácter científico-técnico, tales como La ingeniería industrial española en el siglo XIX (1944), de Alonso Viguera.

Una cuestión que revierte gran importancia para nuestro estudio es conocer la recepción de este género de publicaciones. Para ello recurriremos a un criterio predominante material, relacionado con el precio de las mismas y con el público que disponía de la capacidad económica, e intelectual, para consumirlas. La España de la posguerra estuvo dominada por el hambre y las enfermedades que esta generaba; productos básicos como el pan, los huevos, la leche... eran un lujo al alcance de unos pocos (Arco Blanco, 2006: 241-258). En zonas como Bilbao -que puede servir perfectamente para establecer un modelo comparativo a escala nacional con las grandes urbes- el precio de un kilo de arroz en 1940 era de 1,18 pesetas; el de lentejas 1,34 pesetas o el de un litro de leche 0,78 pesetas $^{41}$. Un simple periódico (por la carencia de papel) como $A B C$ costaba 0,15 céntimos de peseta o la entrada a la película Raza ascendía a 3,35 pesetas. En el presupuesto familiar de la España de 1939 los "gastos diversos" (ocio, principalmente) solo representaba el 7,4 \% del total (Maluquer de Motes, 2013: 59). Los folletos y opúsculos propagandísticos editados e impresos por Blass tenían un precio que podía oscilar entre las dos pesetas (para los de 15 a 20 páginas) llegando incluso a las cinco pesetas, para títulos como Alas germanas sobre Europa. De estos datos podemos concluir que no eran lecturas enfocadas a las clases populares, pues su precio era bastante elevado en comparación a otros productos de la época, no solo los clasificados como "racionados". De hecho, los informes de la embajada alemana especificaban el público de algunos de estos folletos: personalidades

\footnotetext{
${ }^{40}$ Petersen al Ministerio de Exteriores en Berlín, junio de 1942, AA / PA / Botschaft Madrid, Sammlung der Berichte 761.

${ }^{41}$ Estadística de precios e índices de materias primas y productos en España de 1913 a 1941
} 
dirigentes, asociaciones profesionales, universidades, colegios universitarios, bibliotecas, organizaciones de masas, clubes, miembros del consulado y grupos locales del NSDAP ${ }^{42}$. Se trataba, en consecuencia, de una propaganda selectiva por criterios económicos, destinada a grupos de población con unos ingresos altos (por ejemplo, profesiones liberales) y con una formación intelectual elevada, pues en la mayoría de ellos aparecían conceptos teóricos e históricos que requerían estudios superiores y un conocimiento detallado del panorama geopolítico de la época. Se trataba de combinar, como exponía la Sección de Ediciones de la propia VSEP, "el afán informativo y aleccionador" ${ }^{43}$. No obstante, algunos de los folletos encargados a Blass por la embajada debían ser distribuidos gratuitamente. Ese era el caso de Inglaterra a través de la crítica inglesa y, por supuesto, de los anexos de los Boletines de Información y los boletines en $\mathrm{si}^{44}$. A tenor de estos condicionantes y del tipo de lenguaje empleado (retórico, lleno de confrontaciones dialécticas, metáforas...), el "consumidor ideal" serían los universitarios falangistas, de familia acomodada, deseosos de un mayor protagonismo e iniciativa de España en el Nuevo Orden que se estaba forjando. En resumidas cuentas, los herederos de aquellos primeros camisas azules, "los fascinados por el nazismo", como Ramiro Ledesma Ramos u Onésimo Redondo, que a principios de los años treinta, y desde medios como La Libertad o La Conquista del Estado, realizaron una labor de proselitismo ideológico a favor del Tercer Reich en la Península Ibérica (Núñez Seixas, 2016: 28).

La maquinaria propagandística trataría de movilizar a este sector para que colaborase de manera activa en la difusión de sus ideales, mediante protestas antibritánicas, aumentando la presión de la base social del Partido sobre sus dirigentes o arrinconando en el espacio público cualquier manifestación o tendencia de signo aliado. Y, la verdad, es que en algunos casos concretos esta maniobra funcionó. Así, por ejemplo, en Valencia surgió a principios de 1941 dentro de la Falange un grupo clandestino, con conexiones con el consulado, la embajada alemana en Madrid y el NSDAP local, para hacer propaganda pro nazi y amedrentar a elementos anglófilos (Núñez Seixas, 2016: 35).

La decidida colaboración de Joseph Blass en estos planes propagandísticos no pasó desapercibida a las autoridades militares británicas y norteamericanas tras la conclusión de la guerra. Su nombre fue incluido en una lista de colaboradores con la Alemania nazi que requerían ser interrogados para conocer el grado de colaboración con la misma ${ }^{45}$.

\footnotetext{
${ }^{42}$ Heyden-Rynsch al Ministerio de Asuntos Exteriores en Berlín, 8 de Julio de 1941. AA / PA / Botschaft Madrid, Sammlung der Berichte, 761

${ }^{43}$ AGA, Cultura, caja 1388.

${ }^{44}$ Heberlein al Ministerio de Asuntos Exteriores en Berlín, 20 de octubre de 1941. AA / PA / Botschaft Madrid, Sammlung der Berichte, 761. Petersen al Ministerio de Asuntos Exteriores en Berlín, 3 de noviembre de 1941. AA / PA / Botschaft Madrid, Presselenkung 757.

45 NARA, M1933. Reports, letters, cables, and military attaché reports referring to specific Project Safehaven reports, 1944-1945. "List of persons and firms with Axis connections which the American and British".
} 
La consecuencia de todo ello fue negativa para sus bienes empresariales y personales. El régimen franquista, en solidaridad con la Resolución de la VI Conferencia Internacional Financiera y Monetaria de Bretton Woods ${ }^{46}$ (Decreto-ley de 5 de mayo de 1945), bloqueó hasta 1949 todas sus propiedades y las acciones de la sociedad BLASS S.A. Tipográfica ${ }^{47}$. Después de esa fecha prosiguió con su actividad normal hasta su muerte en 1957, siendo homenajeado como "un maestro de las artes gráficas ", creador de una empresa "próspera y admirable" ${ }^{48}$, de la que pocos recordaban su trascendental protagonismo como herramienta de penetración ideológica nazi en los años de la Segunda Guerra Mundial en España.

\section{Reflexiones finales}

Las actividades de la editorial Blass confirman y ponen de relieve no solo la extensión de la red propagandística alemana en España, sino cómo las diversas campañas de propaganda se desarrollaron dentro de un marco caracterizado por la conjunción de tres elementos clave: la iniciativa propagandística de la embajada, la instrumentalización de la colonia alemana y españoles germanófilos, y la activa colaboración de Falange, evidente, en este caso, en la actitud benevolente de la censura y, sobre todo, la complicidad de la VSEP. Estos tres elementos ayudan a comprender cómo esta red creció con tanta rapidez y logró mantenerse hasta el fin de la contienda, a pesar del viraje en política exterior impulsado por Jordana.

Por otra parte, el caso de Blass evidencia asimismo el carácter profundamente anglófobo de la propaganda alemana en España durante la Segunda Guerra Mundial. Al fin y al cabo, esta temática no solo encontraba un terreno abonado en España, y muy particularmente en Falange, sino que proveía a Alemania de un enemigo sencillo y adecuado en un periodo, entre 1939 y junio de 1941, en que el bolchevismo no podía ser objeto de crítica. Además, entroncaba con líneas propagandísticas ya establecidas durante la Primera Guerra Mundial, lo que no puede extrañar ya que Stohrer había sido el encargado de dicha competencia. Por otra parte, a pesar de la política de no

\footnotetext{
${ }^{46}$ Los objetivos perseguidos por el programa safehaven (refugio seguro), incluido en dicha resolución, eran en primer lugar, terminar con las ayudas que, de forma más o menos encubierta, estuvieran prestando los países neutrales al esfuerzo militar nazi. En segunda instancia, inmovilizar en aquellos todos los activos e inversiones alemanas que hubieran recibido. Seguidamente, impedir a toda costa la fuga de bienes y capitales nazis hacia posibles refugios en otros países, y, finalmente, tratar de restituir los bienes robados a sus legítimos dueños y reconstruir las naciones arrasadas por la guerra. Frax Rosales y Matilla Quiza, 2010, pp. 3-4.

${ }^{47}$ AGA, Exteriores, caja 10150. Escrito de Blass Mayer a la Dirección General de Política Económica, Servicio de Blanqueo de Bienes Extranjeros, 19 de agosto de 1948.

48 "Ha muerto Don José Blass, maestro de las Artes Gráficas", $A B C, 16$ de mayo de 1957.
} 
intervención, la actitud británica durante la guerra civil española y los contactos de los exiliados españoles con el gobierno inglés, facilitaban la recepción de estos mensajes precisamente entre el público al que iban dirigidos, muy proclive a aceptarlos. Esta propaganda denota, además, que existía efectivamente una adaptación de los argumentos propagandísticos a distintos países, en contra de lo que han planteado otras obras dedicadas al estudio de la propaganda nazi, asumiendo que la inefectividad de la propaganda se debía a una simple importación de tropos desde Alemania (Schulze Schneider, 1995: 216). Si comparamos los argumentos anglófobos de la propaganda alemana orientados a España con aquellos orientados a Portugal, es fácil observar este fenómeno. Aunque existen, efectivamente, algunas coincidencias como la crítica al bloqueo inglés o la más general hacia Estados Unidos, se observan también importantes diferencias. Así, el mensaje orientado al franquismo sugiere con insistencia que Inglaterra busca desestabilizar el régimen de Franco, mientras que el orientado al salazarismo se centra exclusivamente en la amenaza que supone el imperialismo británico para las colonias portuguesas y en la caducidad de la alianza anglo-portuguesa en una Europa liderada por una Alemania, respetuosa del imperio portugués ${ }^{49}$. También hubo divergencias en el mensaje propagandístico antibritánico que el Tercer Reich proyectó en otras latitudes de África y Asia, en especial en los países árabes, a los que se animaba a defender el derecho a su independencia y a no ser sometidos religiosamente por los aliados en beneficio de la constitución de un macro Estado judío en sus territorios (Herf, 2009: 116, 121-122, 132-133).

Los títulos editados por Blass entre 1939 y 1942 se englobarían mayoritariamente dentro de la literatura propagandística de género más combativo, aquel que únicamente trataba de despertar el odio y desprecio hacia toda acción relacionada con Inglaterra, su forma de vida, su historia y, sobre todo, su actitud en la contienda mundial. Un discurso directo, revestido de una retórica llamativa adecuada al gusto del falangismo español, que crease un estado de opinión totalmente favorable a las tesis nazis. Son pocos los títulos que incidieron en otras temáticas "imprescindibles" para el discurso del Tercer Reich, en especial su política religiosa, su punto argumentativo más débil y controvertido en suelo peninsular. Una excepción la encontramos en La Iglesia católica en Polonia: informe del sacerdote Krawczyk, de 1940, en la que se trataba de demostrar la "total libertad religiosa" en los territorios ocupados por los alemanes en territorio polaco. Folletos y obras de gran relevancia en este sentido como La Iglesia católica en el Reich. Por los fueros de la verdad (1942) ó El cristianismo en el Tercer Reich fueron elaborados directamente por la Embajada (Moreno Cantano, 2013: 61-70) o por la propia VSEP mediante una editorial ficticia como Ediciones Ruta, responsable -entre otras obras- de La hora católica de España, del sacerdote Pedro Cantero, en la que alababa las bondades del régimen de Hitler y la labor evangelizadora de Falange (Ruiz Bautista, 2008: 59).

\footnotetext{
${ }^{49}$ Standardthesen und Richtlinien für die Deutschen Auslandspropaganda, Standardthesen für Portugal und Spanien, 14 Februar 1942, AA / PA / R99474.
} 
Como hemos anotado, Blass no estaba destinada a "construir verdades" sino más bien a "destruir" las del enemigo. Esta operación propagandística que precedió a la gran campaña de 1942 debía allanar el campo ideológico de las élites franquistas (en especial falangistas), y una vez "absorbidas" las mismas, ampliar sus miras al resto de los estratos sociales del pueblo español, conformando una pinza propagandística que hiciese fuerza desde arriba y desde abajo en el escalafón político-social. Solo así se podría entender que diferentes cabeceras periodísticas, como Informaciones, El Alcázar o Arriba, tuviesen el atrevimiento de mostrar (en diciembre de 1941) en primera plana que en el Tercer Reich el catolicismo se desarrollaba con toda normalidad, creando un conflicto diplomático de enorme calado con la Santa Sede, que no daba crédito a lo que se publicaba en la "catolicísima" Nueva España ${ }^{50}$.

Si bien la centralidad de los argumentos antibritánicos se mantuvo durante todo el periodo, el tipo de público al que se orientaba esta propaganda cambió notablemente con el inicio del Gran Plan. Como hemos podido ver, la propaganda alemana que Blass ayudó a diseminar se dirigía a un público acomodado y potencialmente interesado en el desarrollo del conflicto bélico y de la política alemana. Sin embargo, el Gran Plan poseía un público más amplio, que englobaba ahora sí a las clases más populares. Además de recurrir a la propaganda negra, muchas de las publicaciones distribuidas dentro de esta campaña, como los crucigramas, las revistas de aventuras, los pasquines pro-falangistas y los folletos humorísticos, tenían como público objetivo a un sector amplio de la población y estaban escritas en un lenguaje sencillo y directo, caso de títulos como Neptuno o la Colección de los 7. Esta "popularización" de la propaganda nazi se produjo como reacción al incremento de la propaganda aliada en la península y a la evolución desfavorable del conflicto, sobre todo a partir de 1943. Es más, si bien las actividades de la editorial Blass confirman y apuntan el alcance y complejidad de la red de propaganda establecida en España, utilizando diversos impresores y editoriales por todo el territorio español, destacan también la preocupación de la embajada ante el aumento de la propaganda aliada, que llevó a la embajada alemana a abrir editoriales encubiertamente propias en 1942, como ya hemos señalado. La apertura de estas editoriales coincide con un periodo en el que el número de obras publicadas por la editorial Blass comienza a disminuir, lo que indica posiblemente que o bien la labor de editoriales como Blass y Rubiños ya no era suficiente, o que la embajada prefería mantener un control directo sobre estas publicaciones $y$, posiblemente, abaratar costes.

Joseph Blass se yergue además como exponente de un amplio sector de la colonia alemana asentada en España, que se sentía tanto español como alemán, pero que ante el fin de la guerra no dudaba en poner su españolismo y, sobre todo, su franquismo como prueba de su inocencia ante las políticas desnazificadoras de los Aliados (Messenger, 2014). Un fenómeno que si bien denotaba, en muchos casos, cierto

\footnotetext{
${ }^{50}$ Archivo del Ministerio de Asuntos Exteriores, R. 3463/19. Apuntes para S.E., 23 de diciembre de 1941.
} 
oportunismo y, por supuesto, afán de supervivencia, indicaba también cuáles eran las bases, para mucha gente, de la sintonía ideológica entre el nazismo y el franquismo.

En conjunto, el estudio de la editorial Blass confirma aspectos clave del funcionamiento, naturaleza y extensión de la propaganda nazi en España y, lo que es más importante, arroja luz sobre el tipo de "consumidores" al que iba dirigida, destacando además el momento en que este público objetivo comenzó a ampliarse. No obstante, futuros estudios, de esta y otras editoriales que sirvieron como cauce de propaganda para la Embajada alemana, podrán contribuir a un mejor conocimiento de la extensión y funcionamiento de la red de propaganda nazi en la Península Ibérica, el papel que en ello jugó la colonia alemana y, no menos importante, si estas editoriales llegaron a funcionar también como canales para la difusión de las ideas nacional-socialistas en América Latina.

\section{Referencias bibliográficas}

ALBES, J. (1996): Worte wie Wafen: Die deutsche Propaganda in Spanien während des Ersten Weltkrieges, Essen, Klartext.

ÁLVAREZ CHILLIDA, G. (2002): El antisemitismo en España. La imagen del judío (18122002), Madrid, Marcial Pons.

ARCO BLANCO, M. A. (2006): "Morir de hambre. Autarquía, escasez y enfermedad en la España del primer franquismo", en Pasado y memoria. Revista de Historia Contemporánea, n.오, pp. 241-258.

BERNAL MARTíNEZ, I. (2007): "Libros, bibliotecas y propaganda nazi en el primer franquismo: las exposiciones del libro alemán", Hispania Nova, n.o 7, pp. 1-31.

COLLADO SEIDEL, C. (2006): Der Spanische Bürgerkrieg. Geschichte eines europäischen Konflikts, München, Beck.

DANKELMANN, O. (1969): “Der faschistische Grosse Plan. Zur Propaganda-tätigkeit des deutschen Imperialismus in Spanien während des zweiten Weltjkrieges", Zeitschriften für Geschichtswissenschaft, n.o 5, pp. 601-610.

DE LA HERA MARTÍNEZ, J. (2002): La política cultural de Alemania en el período de entreguerras, Madrid, CSIC.

DELGADO GÓMEZ-ESCALONILLA, L. (1992): Imperio de papel: acción cultural y política exterior durante el primer franquismo, Madrid, CSIC. 
EMBAJADA ALEMANA (1940): El calvario de Chipre, Madrid, Blass.

Estadística de precios e índices de materias primas y productos en España de 1913 a 1941, Bilbao, Publicaciones de la Liga Vizcaína de Productores.

EVANS, R. J. (2010): The Third Reich at War: 1939-1945, Westminster, Penguin Books.

FERNÁNDEZ-LONGORIA, M. (2007): La diplomacia británica y el primer franquismo: las relaciones internacionales hispano-británicas durante la Segunda Guerra Mundial, Tesis doctoral, Madrid, UNED

FONTES, I. y MENÉNDEZ, M. A. (2004): El Parlamento de papel: las revistas españolas en la transición democrática, Madrid, Asociación de la Prensa de Madrid.

FORCZYK, R. (2016): We march against England. Operation Sea Lion, 1940-1941, New York, Osprey Publishing.

FRANK, R. (1940): El dominio inglés en La India, Madrid, Blass.

FRAX ROSALES, E. y MATILLA QUIZA, Mạ. J. (2010): “Los seguros negocios del franquismo: el proceso de bloqueo, expropiación y liquidación de las compañías de seguros con capital alemán (1945-1952)", en Jerònia Pons Pons y María Ángeles Pons Brías, Investigaciones históricas sobre el seguro español, Madrid, Fundación Mapfre, pp. 257-258.

GARCÍA PÉREZ, R. (1994): Franquismo y Tercer Reich: las relaciones económicas hispanoalemanas durante la segunda guerra mundial, Madrid, Centro de Estudios Constitucionales.

HAYWARD, J. (2016): Burn the Sea. Flame Warfare, Black Propaganda and the Nazi Plan to Invade England, Gloucestershire, The History Press.

JANUÉ I MIRET, M. (2001): “Relaciones culturales en el Nuevo orden: la Alemania nazi y la España de Franco", en Hispania, vol. LXXV, no. 251, septiembre-diciembre, pp. 805-832.

KIESEWETTER, B. (1941): La liquidación del prestigio económico mundial de Inglaterra en la guerra, Madrid, Blass.

LEITZ, C. (1996): Economic Relations Between Nazi Germany and Franco's Spain, 19361945, New York, Oxford University Press.

LONGERICH, P. (1987): Propagandisten im krieg. Die Presseabteilung des Auswärtigen Amt unter Ribbentrop, München, R. Oldenbourg Verlag. 
MALUQUER DE MOTES, J. (2013): "La inflación en España. Un índice de precios de consumo, 1830-2012", en Estudios de Historia Económica, n.o 64, Madrid, Banco de España.

MÁRQUINA, A. (2014): "La etapa de Ramón Suñer en el Ministerio de Asuntos Exteriores: España se convierte en un país del Eje y pierde la neutralidad", en UNISCI Discussion Papers, n.o 36, pp. 99-121.

MARTÍNEZ MARTíN, J. A. (2001): Historia de la edición en España (1836-1936), Madrid, Marcial Pons.

MARTÍNEZ RUS, A. (2014): La persecución del libro: hogueras, infiernos y buenas lecturas (1936-1951), Gijón, Trea.

MESSENGER, D. (2014): Hunting Nazis in Franco's Spain, Baton Rouge, Louisiana State University Press.

MORADIELLOS, E. (2005): Franco frente a Churchill, Barcelona, Península.

MORENO CANTANO, A. C. (2008): Los servicios de Prensa Extranjera en el primer franquismo, Tesis doctoral, Universidad de Alcalá de Henares.

- (2013): "Literatura de propaganda religiosa en España en tiempos de guerra, 1936-1945", en Antonio César Moreno Cantano (coord.), Cruzados de Franco. Propaganda y diplomacia en tiempos de guerra (1936-1945), Gijón, Trea, pp. 3570.

- (2016): Tiempo de mentiras. El control de la prensa extranjera en España durante el primer franquismo (1936-1945), Sarrión, Muñoz Moya Editores.

NÚÑEZ SEIXAS, X. M. (2016): Camarada invierno: experiencia y memoria de la División Azul, Barcelona, Crítica.

OLMOS, V. (2002): Historia del ABC, Barcelona, Plaza \& Janés.

PEÑALBA-SOTORRÍO, M. (2012): «German Propaganda in Francoist Spain: Diplomatic Information Bulletins as a Primary Tool of Nazi Propaganda», Bulletin for Spanish and Portuguese Historical Studies, 37, 1.

- (2016): "Hans Lazar, Master of Propaganda", Seminario de Historia, Fundación José Ortega y Gasste-Gregorio Marañón, pp. 1-20.

- (2018): "Beyond The War: Nazi Propaganda Aims in Spain during the Second World War", Journal of Contemporary History, pp. 1-25.

- (inédita): “La II Guerra Mundial en el diario Arriba o cómo la propaganda sirve a la política", comunicación presentada en International Conference, Theatres of 
War: War and Post-war Experiences in European Societies (1895-1953), Universitat Autònoma de Barcelona, (Barcelona, 2015).

PIZARROSO QUINTERO, A. (2009): Diplomáticos, propagandistas y espías: Estados Unidos y España en la Segunda Guerra Mundial. Información y propaganda, Madrid, CSIC.

ROS AGUDO, M. (2002): La guerra secreta de Franco (1939-1945), Barcelona, Crítica.

(2008): La gran tentación: Franco, el Imperio colonial y los planes de intervención en la Segunda Guerra Mundial, Barcelona, Styria.

RUBIN, B. y SCHWANITZ, W. G. (2014): Nazis, Islamists and the Making of the Modern Middle East, New Haven, Yale University Press.

RUHL, K.-J. (1986): Franco, Falange y "Tercer Reich": España en la Segunda Guerra Mundial, Madrid, Akal.

RUIZ BAUTISTA, E. (2005): Los señores del libro: propagandistas, censores y bibliotecarios en el primer franquismo, Gijón, Trea.

-(2008): Tiempo de censura. La represión editorial durante el franquismo, Gijón, Trea.

- y BARRUSO BARÉS, Pedro (2011), "La propaganda alemana en España durante la Segunda Guerra Mundial", en Antonio César Moreno Cantano (coord.), El ocaso de la verdad. Propaganda y prensa exterior en la España franquista, Gijón, Trea, pp. 191-214.

SCHULZE SCHNEIDER, I. (1994): “La propaganda alemana en España, 1942-1944", Espacio, tiempo y forma, Serie V, Historia Contemporánea, n.ㅇ 7, pp. 371-386.

- (1995): “Éxitos y fracasos de la propaganda alemana en España: 1939-1944", Mélanges de la Casa de Velázquez, n.o 31, pp. 197-218.

- (2004): “Alemania y la guerra civil española: información y propaganda”, Spagna contemporanea, 26 , pp. 57-84.

SEVILLANO CALERO, F. (2000): Ecos de papel: la opinión de los españoles en la época de Franco, Madrid, Biblioteca Nueva.

SPECTATOR (Pseudónimo de Alberto Martín Fernández) (1941): Alas germanas sobre Europa, Madrid, Blass.

STROBL, G. (2000): The Germanic Isle: Nazi Perceptions of Britain, Cambridge, Cambridge University Press.

TAUBE, M. (1944): "The Publishing Activities of the Deutsche Informationsstelle", Quaterly Journal of Current Acquisitions, vol. 2, no 1, pp. 86-90. 
VIÑAS, Á. (2016): Sobornos. De cómo Churchill y March compraron a los generales de Franco, Barcelona, Crítica.

WADDINGTON, L. (2007): "The Anti-Komintern and Nazi Anti-Bolshevik Propaganda in the 1930s", Journal of Contemporary History, Vol. 42, n.o 4, pp. 573-594.

- (2012): Hitler's crusade: bolshevism, the Jews, and the myth of conspiracy, London, I.B. Tauris.

WHEALEY, R. H. (1989): Hitler and Spain: The Nazi Role in the Spanish Civil War, 19361939, Kentucky, Kentucky University Press. 